\title{
EXIT Chart Optimization of Turbo-FSK: Application to Low Power Wide Area Networks
}

\author{
Yoann ROTH ${ }^{1}$, Jean-Baptiste DoRÉ ${ }^{1}$, Laurent $\operatorname{Ros}^{2}$ and Vincent BERG ${ }^{1}$ \\ ${ }^{1}$ CEA, LETI, MINATEC Campus, F-38054 Grenoble, France \\ ${ }^{2}$ Univ. Grenoble Alpes, GIPSA-Lab, F-38000 Grenoble, France \\ \{yoann.roth, jean-baptiste.dore, vincent.berg\}@cea.fr, laurent.ros@gipsa-lab.grenoble-inp.fr
}

\begin{abstract}
Low Power Wide Area (LPWA) networks aim at connecting low cost devices with high constraints on the link budget. Focusing on the physical layer, the combination of Frequency Shift Keying (FSK) modulation and coding in the same process has been shown efficient when associated to a turbo receiver. This paper considers the optimization of Turbo-FSK parameters for the LPWA context using the EXtrinsic Information Transfer (EXIT) Chart analysis. After adapting the metric to the FSK waveforms, EXIT chart performance results are extended and compared to short packet lengths using extensive Bit Error Rate (BER) computations.
\end{abstract}

Keywords-Low Power Wide Area, LPWA, Internet of Things, PHY-layer, FSK, Turbo Code, Low Rate, Low SNR, Machine-toMachine, EXIT Chart

\section{INTRODUCTION}

Machine-to-Machine (M2M) type communication is expanding, and the potential market for low-powered wireless solutions in the Internet-of-Things (IoT) is expected to grow exponentially [1]. In this Low Power Wide Area (LPWA) networks, terminals are expected to be low cost, to have a low power consumption, and to be able to communicate at long range. A link budget improvement of $15-20 \mathrm{~dB}$ in comparison to existing cellular technologies is also expected [2]. These critical issues set major constraints on the physical layer, as complexity at the terminal level and sensitivity working levels must be kept minimum.

A commonly employed technique designed to reach low sensitivity levels is the well-known repetition scheme (spread spectrum). The information data is transmitted $\lambda$ times in order to give a processing gain of $10 \log _{10}(\lambda)$ in Signal-toNoise Ratio (SNR) at the receiver, but the spectral efficiency is divided by $\lambda$. This technique improves the sensitivity level when a fixed bandwidth is considered, but does not improve the energy efficiency. Many systems rely on this scheme and so does Direct Sequence Spread Spectrum (DSSS). The IEEE standard 802.15.4k [3] has been specifically designed for low power infrastructure monitoring using the DSSS technique. A natural choice for low data rate and low power applications is the use of $M$-ary orthogonal modulation. For this specific type of non-linear modulation, increasing the alphabet size $M$ gives a gain in energy efficiency while reducing the spectral efficiency [4]. This property can be extended to Orthogonal Sequence Spread Spectrum (OSSS), and is purportedly used by the LoRa technology [5], as suggested in [6]. This solution aims at long range communication for the IoT.

The ultimate Shannon's limit [7] gives the maximum transmission rate with arbitrarily small bit-error probability, for a given Signal-to-Noise Ratio (SNR) and a given bandwidth. This limit defines the minimum required $E_{b} / N_{0}$ for a reliable communication as an increasing function of the spectral efficiency, and it can be shown that this value is lowerbounded by the ultimate limit $E_{b} / N_{0}=-1.59 \mathrm{~dB}$. The previously mentioned schemes have some significant limitations when this limit is considered. Orthogonal modulations are shown to reach the ultimate limit, but an infinite size of alphabet is required, thus reducing the spectral efficiency to 0. Alternatively, DSSS does not improve the energy efficiency, as the $E_{b} / N_{0}$ is not affected by spreading the information, and leads to potential energy waste. Our previous work [8] already highlighted this issue, and suggested that in the LPWA context, more elaborate decoding schemes, such as turbo processing [9], should be considered as efficient alternatives. These schemes indeed offer high performance at the expense of complexity increase at the receiver side, but low power consumption still can be ensured at the terminal side.

The use of orthogonal alphabet combined with turbo decoding was first proposed with the introduction of the TurboHadamard code presented in [10], where binary Hadamard codes are studied. In [8], we adapted the scheme and decoder computations to $M$-ary Frequency-Shift-Keying (FSK) modulation, and introduced the technique in the LPWA context. FSK modulation has already been considered for low power wireless networks as its constant envelope property provides a power efficient solution. This modulation is widely used for monitoring applications, for which power efficient and low cost off-the-shelf optimized chips are available [11]. A comparison with standard LPWA solutions is done in [12], showing the potential of turbo processing for the IoT context. Limitations of the Turbo-Hadamard scheme have been studied in [13], using the EXtrinsic Information Transfer (EXIT) Chart analysis [14], [15]. The emphasis has primarily been on finding the minimum pinch-off value for every possible set of parameters. Pinch-off value is defined as the $E_{b} / N_{0}$ value for which the decoder can recover without error the information word after a certain number of iterations. The Turbo-Hadamard scheme was shown to approach Shannon's limit by only $0.29 \mathrm{~dB}$.

In this paper, we propose to optimize the energy efficiency of the Turbo-FSK scheme, using the EXIT Chart analysis. The computation proposed by [13] for Turbo-Hadamard does not directly apply and adaptations are derived for the FSK case. Then, we perform simulations to find the pinch-off values for every set of parameters. The analysis assumes perfect decorrelation between the different sent versions, and this hypothesis tends to be valid when a large information block size is considered. For LPWA, short length packets are expected, we therefore propose to perform extensive Bit-ErrorRate (BER) simulation to confirm if the behavior predicted by the EXIT Charts can be valid to short packet sizes.

The paper is organized as follows. The Turbo-FSK ar- 


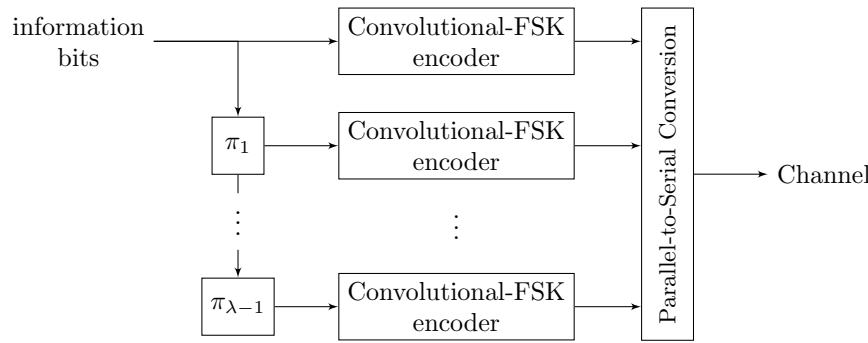

Fig. 1. The Turbo-FSK transmitter.

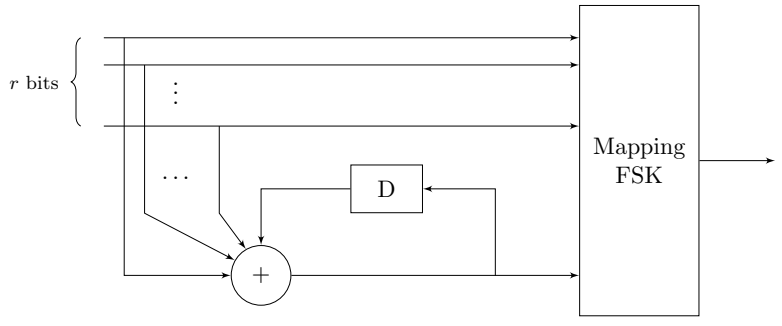

Fig. 2. The Convolutional-FSK encoder.

chitecture from [8] is briefly presented in Section II. EXIT Chart computation is presented in Section III, while Section IV presents the optimization process proposed. Section V concludes the paper.

\section{SySTEM MODEL}

In this section, we will briefly introduce the Turbo-FSK scheme presented in [8].

\section{A. Transmitter}

Transmitter architecture is given in Figure 1. The structure is composed of $\lambda$ stages, each one encoding a differently interleaved version of the input bits. Information bits are gathered into $P$ groups of $r$ bits, and Convolutional-FSK encoding is applied, as described in Figure 2. For every block of $r$ bits, a single parity bit is added. The parity bit consists of the modulo- 2 sum of the $r$ input bits and the parity bit of the previous block. The resulting $r+1$ bits are then mapped to a complex codeword of the FSK alphabet, whose size is $M=2^{r+1}$. Thanks to the accumulation, every consecutive symbol is related to the previous one. The output of each encoder is then a set of $P$ FSK codewords: this scheme mixes coding and modulation in the same process. A Parallelto-Serial Conversion is then done in order to transmit the FSK codewords through the channel. The normalized spectral efficiency is defined by ${ }^{1}$

$$
\eta=\frac{\log _{2}(M)-1}{\lambda M}
$$

and expressed in bits/s/Hz.

\section{B. Channel}

We use a discrete-time model and consider an Additive White Gaussian Noise (AWGN) channel, where the output is defined as

$$
\boldsymbol{y}=\boldsymbol{x}+\boldsymbol{n},
$$

\footnotetext{
${ }^{1}$ The trellis termination has been omitted. One extra FSK codeword must be emitted in order to close the trellis, thus slightly reducing the spectral efficiency.
}

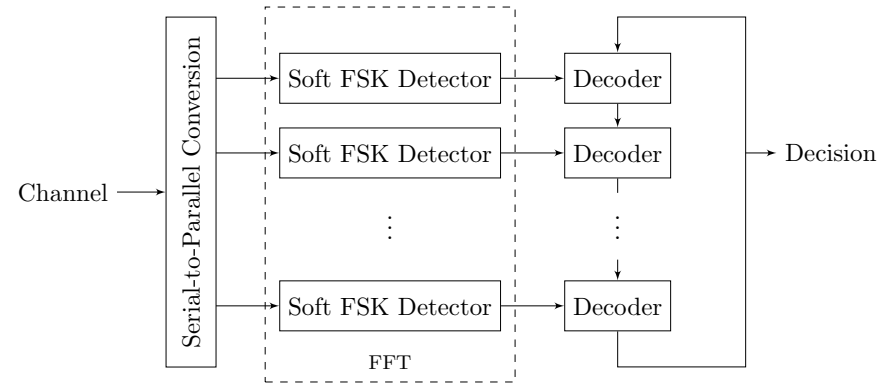

Fig. 3. The Turbo-FSK receiver.

with $\boldsymbol{x}$ is the emitted signal. $\boldsymbol{n}$ is the complex additive white Gaussian noise vector, with variance $\sigma^{2} . \boldsymbol{x}, \boldsymbol{y}$ and $\boldsymbol{n}$ are complex vectors of size $M \lambda(P+1)$.

\section{Receiver}

The receiver, depicted in Figure 3, includes a Serial-toParallel Conversion, to reconstruct the $\lambda$ stages emitted at the first place. A soft FSK detector is used to determine the probabilities of each possible codeword. This step can be performed using the Fast Fourier Transform (FFT) algorithm. These probabilities are then fed to the decoder, which uses them as channel observation, while output of the other decoders will be used as a priori information. BCJR algorithm [16] is used to decode the trellis, and the A Posteriori Probabilities (APP) of the information bits are computed. Orthogonality of the transmitted codewords is one of the key features of this scheme, which offers a great performance gain compared to simple repetition schemes. For detailed explanations about the receiver's computations, reader is invited to refer to [8].

\section{EXIT CHART ANALYSIS}

The EXIT Chart analysis was first introduced in [14] as a way to predict the threshold $E_{b} / N_{0}$ for which the BER drops significantly (the "waterfall" region). The analysis was performed on standard Parallel Concatenated Convolutional Codes (PCCC), where, at the receiving side, two decoders exchange information in an iterative way. During the process, the information bits are represented by Log Likelihood Ratio (LLR), defined as

$$
L(b)=\log \frac{p(b=1)}{p(b=0)},
$$

where $p(b=1)$ (resp. $p(b=0))$ is the probability that the bit $b$ equals 1 (resp. 0$)$. The Turbo-Hadamard code EXIT Chart analysis has been realized in [13].

\section{A. EXIT Chart computation for the FSK case}

While the PCCC or Turbo-Hadamard code are systematic codes, the suggested architecture in [8] uses FSK waveforms as codeword. The process to compute the extrinsic information is depicted Figure 4. The observation from the channel are directly converted to codewords probabilities, represented in blue on the graph. The BCJR decodes the trellis and gives the output LLRs on the information bits $L_{\mathrm{APP}}$, that can be expressed

$$
L_{\mathrm{APP}}=L_{A}+L_{\mathrm{ch}}+L_{E}
$$




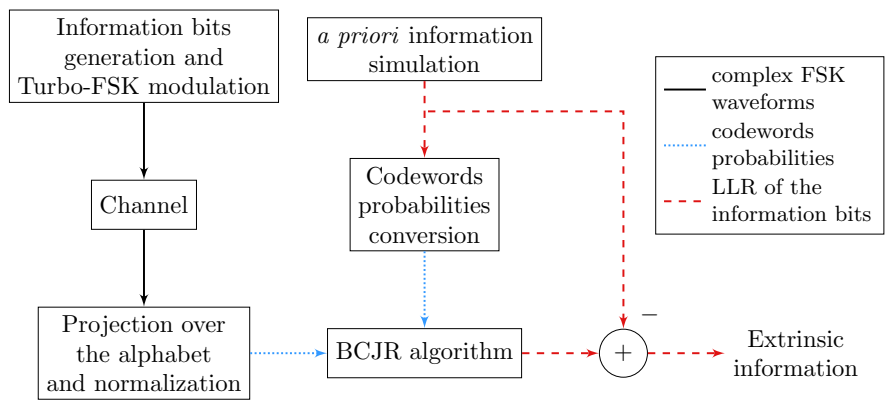

Fig. 4. The Turbo-FSK EXIT extrinsic information extraction.

where $L_{A}$ is the output of the a priori information simulation block, or the a priori LLR used by the BCJR algorithm. $L_{E}$ is the extrinsic information created by the algorithm, and $L_{\mathrm{ch}}$ is the information from the channel. The FSK codewords being non-systematic, it is not possible to extract the LLR from the channel as it is usually done for systematic codes; hence the value $L_{\mathrm{ch}}$ cannot be properly computed ${ }^{2}$. It is however possible to subtract the a priori LLR $L_{A}$, and to refer to the results as the extrinsic information. The influence of this approximation will be discussed later.

\section{B. Multidimensional EXIT Chart analysis}

The extension of the EXIT Chart analysis to multidimensional codes, where more than two decoders are used, has been studied in [15]. If $\lambda$ decoders are considered, the representation of the exchanges of information should be represented in $\lambda$ dimensions. Tracking directly the exchanges of information becomes impossible for $\lambda>3$ (as it would require more than 3 dimensions), but in the case where all decoders are the same, a two-dimensional projection may be computed. If the projection does not intersect with the line going from $(0,0)$ to $(1,1)$, it indicates that convergence toward the maximum of mutual information is possible. The a priori information fed to one decoder is the sum of the extrinsic information of the $(\lambda-1)$ other decoders. To obtain the projection, the formula of the a priori mutual information $I_{A}$ must be modified. Using the function $J$ as defined in [14], it can be expressed

$$
I_{A}=J\left(\sqrt{\lambda-1} \sigma_{A}\right),
$$

where $\sigma_{A}$ is the standard deviation of the a priori model, chosen as Gaussian as suggested in [14]. To compute the extrinsic mutual information $I_{E}$, we use the general formula where density probability functions need to be integrated.

\section{Comparison with the Turbo-Hadamard case}

The EXIT Chart analysis done for the Turbo-Hadamard in [13] considers both a systematic and multidimensional code. We chose to study the impact of: (1) the implication of dealing with non-systematic codes and (2) the non-binary codewords inducing the modification of the EXIT Chart computation previously presented. For (1), we perform simulation of the EXIT Chart of the Turbo-Hadamard case for which the systematic bits are repeated. In comparison to the case where systematic bits are not repeated, the process of repeating them changes the observed channel on those bits. We refer

\footnotetext{
${ }^{2}$ Decoding only the FSK modulation would still include the extrinsic information of the orthogonal code itself.
}

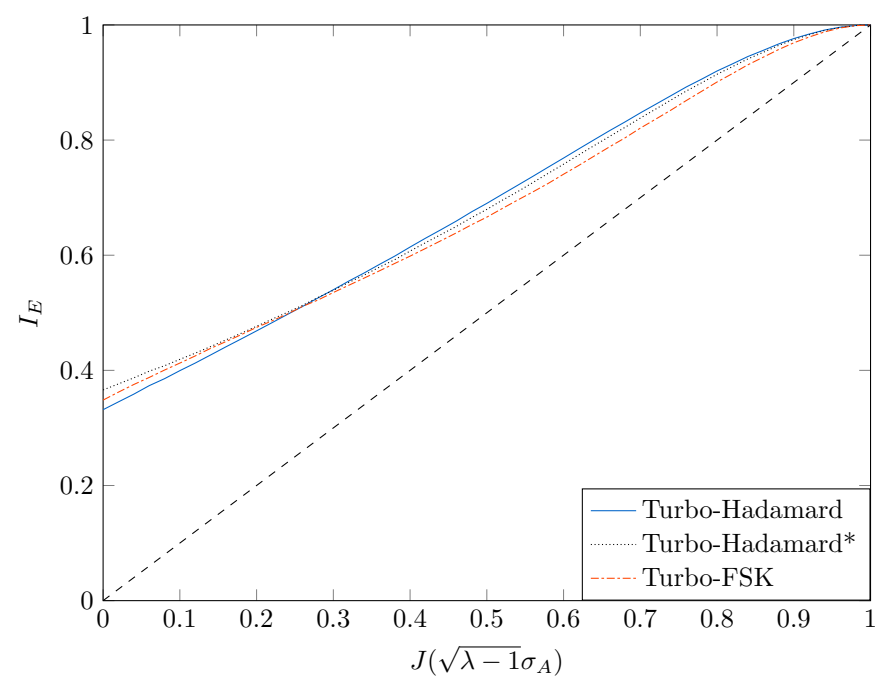

Fig. 5. Comparison of the different schemes for the same parameters $M=$ $16, \lambda=3$. The information block size was set to $N=100000$, and channel setting is $E_{b} / N_{0}=2 \mathrm{~dB}$.

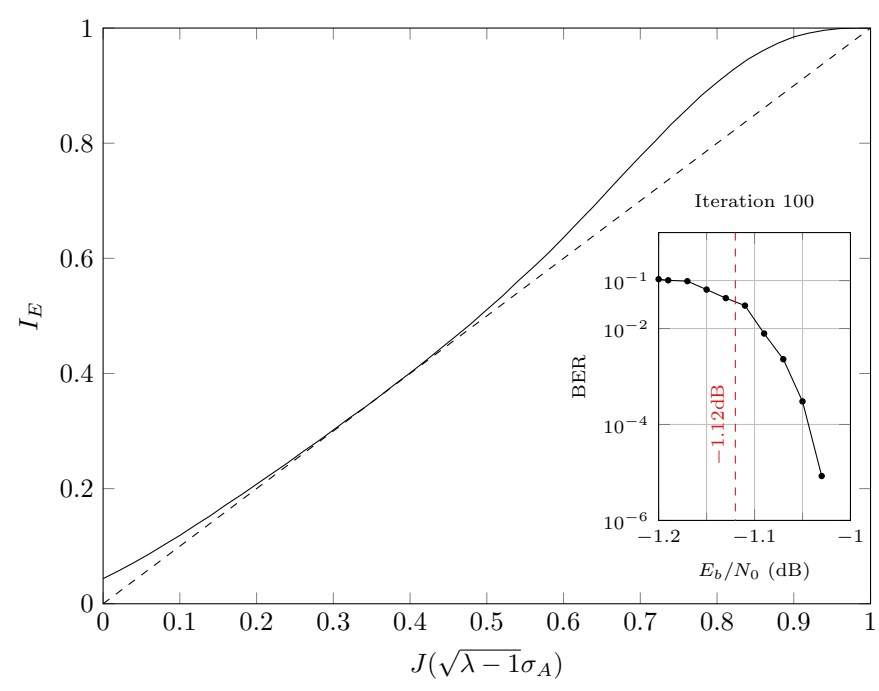

Fig. 6. EXIT Chart and BER performance for the Turbo-FSK scheme with parameters $M=128, \lambda=4$. EXIT Chart is computed for $E_{b} / N_{0}$ $=-1.12 \mathrm{~dB}$, with $N=100000$. BER performance is computed using an information block size of $N=100000$ and the MAP algorithm.

to this scheme as Turbo-Hadamard*. For (2), we compare for the same parameters the EXIT Chart of the Turbo-FSK decoder with the equivalent Turbo-Hadamard decoder. The EXIT Charts of the three different decoders are depicted Figure 5. The EXIT Charts are very close, but these non-similarities will induce small differences in the pinch-off values of each scheme.

\section{Comparison with the BER performance}

In order to check if the EXIT Chart analysis predicts the waterfall region, we look for the pinch-off value of a particular case and compute the Bit-Error-Rate (BER) for the same case with a large block size of information bit $(N=100000)$. The case $M=128$ and $\lambda=4$ is selected. The pinch-off value found is $E_{b} / N_{0}=-1.12 \mathrm{~dB}$. The EXIT Chart for this $E_{b} / N_{0}$ and the BER around this value are depicted Figure 6. After 


\begin{tabular}{c|cccccccc}
\hline$\lambda$ & $M$ & & & & & & \\
\end{tabular}

TABLE I. PINCHOFF VALUES IN $E_{b} / N_{0}$ FOR EVERY $M$ AND $\lambda$ TESTED. EXIT CHART ARE COMPUTED USING AN INFORMATION BLOCK SIZE $N=100000$. BEST VALUE FOR EACH $M$ IS DENOTED WITH A SYMBOL *, AND BEST COUPLE OF PARAMETERS IS IN BOLD.

100 decoder iterations, the waterfall appears around $-1.1 \mathrm{~dB}$, very close to the value predicted by the EXIT Chart.

\section{PARAMETERS OPTIMIZATION}

The Turbo-FSK scheme offers two adjustable parameters that can be optimized for better energy efficiency. Using the EXIT Chart analysis previously introduced and BER computations, this section is dedicated to the exhaustive search for sets of parameters $(M, \lambda)$ as close as possible of the Shannon's limit. For all simulations, coherent detection of the FSK and perfect synchronization are assumed. MAP algorithm without any approximation is considered.

\section{A. Optimization using the EXIT Chart analysis}

As the EXIT Chart satisfyingly predicts the waterfall, this tool can be used to determine the energy efficiency of the Turbo-FSK scheme for large information block sizes. An exhaustive search was realized on a large number of sets of $(M, \lambda)$. Alphabet size selected varies from $M=16$ to $M=2048$, and repetition factor is chosen between 2 and 6. A block size $N=100000$ is used. The pinch-off values obtained are presented Table $\mathrm{I}^{3}$. The minimum $E_{b} / N_{0}$ value for each alphabet size is denoted by a star ${ }^{*}$. The couple of parameters with the lowest pinch-off value is $(512,3)$, only $0.29 \mathrm{~dB}$ away from Shannon's limit. For the Turbo-Hadamard case, an equivalent result is obtained in [13], despite the differences between the codes. The performance depending on the value of $\lambda$ seems unpredictable and the exhaustive search enables the optimization of the parameters.

\section{B. Optimization based on BER computations}

As previously mentioned, the EXIT Chart analysis assumes perfect independence between the different interleaved information messages of the decoder and a large size of information block guarantees this property. Considering the LPWA context, small block sizes are expected. In order to analyze the influence of the block size and the interleaver, we perform extensive BER computation. For every set of parameter, we search the $E_{b} / N_{0}$ value for which BER reach $10^{-4}$. Block size is set to $N=1000$, and the decoder performs 10 iterations. A random interleaver is used. Values obtained are depicted Table II. For the best case, the gap to Shannon's limit is now $1.35 \mathrm{~dB}$. The corresponding set of parameters is $(1024,3)$. The performance of the best set found with EXIT Chart analysis is

\footnotetext{
${ }^{3}$ For $\lambda=2$, values may be overestimated due to the fact that the EXIT Charts cross the line $(0,0) \rightarrow(1,1)$ very close to the point $(1,1)$.
}

only $0.02 \mathrm{~dB}$ away from this set. Performance improvements may be achieved through the use of an optimized interleaving function.

\section{Comparison to the channel capacity}

The spectral efficiency of the Turbo-FSK, as defined in (1), is different for every set of parameters. Considering the normalized spectral efficiency versus the energy efficiency, the values previously obtained can be compared to the ultimate Shannon's limit [7], defined as

$$
E_{b} / N_{0} \geq \frac{2^{\eta}-1}{\eta}
$$

The result is depicted in Figure 7, where for each alphabet size, $\lambda$ values from 3 to 6 are represented. As $\lambda$ is increased, the normalized spectral efficiency is lowered; the point with the higher $\eta$ is $\lambda=3$, then 4 and so on. The figure clearly shows the optimum value for the parameters, for both the EXIT Chart analysis and the BER computations for small block sizes. The general trend obtained with the EXIT Chart seems to be valid when the block size is shortened. The performance loss incurred by the block size reduction is $1 \mathrm{~dB}$ on average (between 0.8 and $1.25 \mathrm{~dB}$ ).

\section{CONCLUSION}

Within the LPWA context, the energy efficiency of the transmission scheme is a major issue. The Turbo-FSK scheme is a way to achieve very low levels of sensitivity, exploiting repetition, orthogonal modulation, and a complex decoding algorithm, while ensuring a low consumption transmitter. Optimization using both EXIT Chart analysis and BER computations was performed. The best set of parameters is shown to be only $0.29 \mathrm{~dB}$ away from the channel capacity for large block size, and $1.35 \mathrm{~dB}$ away for a block size of 1000 bits which is compatible given considerations for the M2M context. A highly reliable communication can be achieved with a nearlyoptimal use of the energy resource. However, reducing the sensitivity also implies to work at very low levels of Signal-toNoise Ratios, for which detection and synchronization become problematic. Repetition in the preamble offers here again a solution, but such a simple scheme will cause severe $E_{b} / N_{0}$ degradation (e.g a preamble as long as the data will induce a $3 \mathrm{~dB}$ loss of $\left.E_{b} / N_{0}\right)$. More elaborate algorithms for synchronization can be conceived to settle this issue. 


\begin{tabular}{c|cccccccc}
\hline & $M$ & & & & & & \\
2 & 16 & 32 & 64 & 128 & 256 & 512 & 1024 & 2048 \\
\hline 2 & 3.00 & 2.62 & 2.09 & 1.64 & 1.25 & 0.91 & 0.57 & 0.31 \\
\hline 3 & 1.40 & 0.89 & 0.48 & 0.14 & -0.13 & $-0.22^{*}$ & $-\mathbf{0 . 2 4 ^ { * }}$ & $0.00^{*}$ \\
\hline 4 & 0.95 & 0.49 & 0.13 & $-0.10^{*}$ & $-0.17^{*}$ & 0.02 & 0.35 & 0.52 \\
\hline 5 & 0.84 & 0.40 & $0.08^{*}$ & -0.04 & 0.08 & 0.35 & 0.78 & 1.07 \\
\hline 6 & $0.74^{*}$ & $0.37^{*}$ & 0.13 & 0.04 & 0.35 & 0.73 & 1.10 & 1.46
\end{tabular}

TABLE II. $\quad E_{b} / N_{0}$ VALUES FOR WHICH BER IS $10^{-4}$, FOR EVERY $M$ AND $\lambda$. BER IS COMPUTED FOR AN INFORMATION BLOCK SIZE $N=1000$, MAP ALGORITHM IS USED, AND 10 ITERATIONS ARE PERFORMED. BEST VALUE FOR EACH $M$ IS DENOTED WITH A SYMBOL *, AND BEST COUPLE OF PARAMETERS IS IN BOLD.
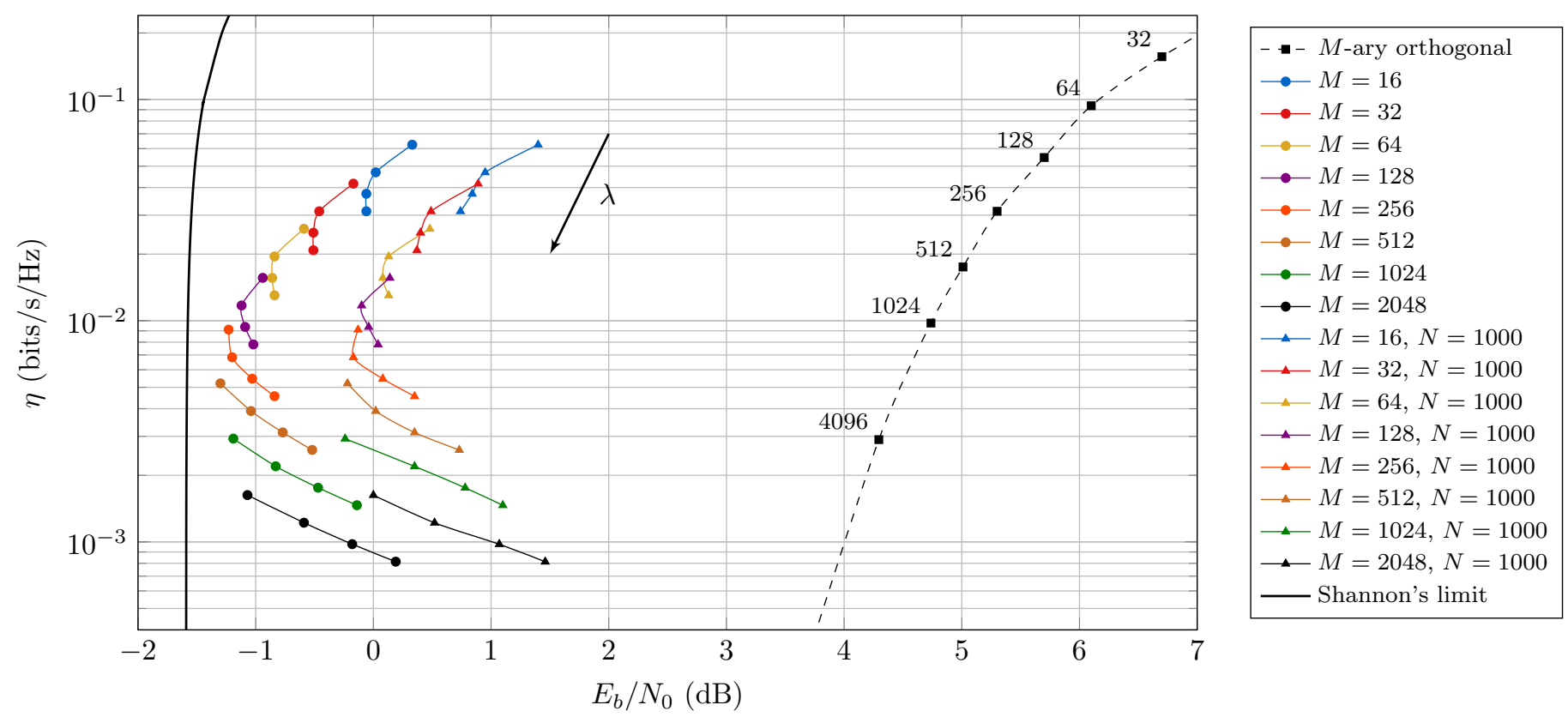

Fig. 7. Values from Table I (EXIT Chart analysis with $N=100000$ ) and Table II (BER simulation for $N=1000$ ) in the plot normalized spectral efficiency versus energy efficiency, for $\lambda$ values going from 3 to 6.

\section{REFERENCES}

[1] T. Rebbeck, M. Mackenzie, and N. Afonso, "Low-powered wireless solutions have the potential to increase the M2M market by over 3 billion connections," Analysys Mason, Sept 2014.

[2] "LTE-M - Optimizing LTE for the Internet of Things," Nokia White Papers, 2015.

[3] "802.15.4k: Low-Rate Wireless Personal Area Networks (LR-WPANs) Amendment 5: Physical Layer Specifications for Low Energy, Critical Infrastructure Monitoring Networks." IEEE Standard for Local and metropolitan area networks, pp. 1-149, Aug 2013.

[4] J. Proakis, Digital Communications 3rd Edition, ser. Communications and signal processing. McGraw-Hill, 1995.

[5] "LoRa Alliance," https://www.lora-alliance.org/, accessed: June 16, 2016.

[6] O. Seller and N. Sornin, "Low power long range transmitter," US Patent 20140219329 Al, Aug 2014.

[7] C. Shannon, "A mathematical theory of communication," The Bell System Technical Journal, vol. 27, no. 3, pp. 379-423, July 1948.

[8] Y. Roth, J.-B. Dore, L. Ros, and V. Berg, "Turbo-FSK: A New Uplink Scheme for Low Power Wide Area Networks," in 2015 IEEE 16th International Workshop on Signal Processing Advances in Wireless Communications (SPAWC), June 2015, pp. 81-85.

[9] C. Berrou, A. Glavieux, and P. Thitimajshima, "Near Shannon limit error-correcting coding and decoding: Turbo-codes. 1," in IEEE International Conference on Communications (ICC). Geneva., vol. 2, May 1993, pp. 1064-1070.

[10] L. Ping, W. Leung, and K. Y. Wu, "Low-rate turbo-Hadamard codes," IEEE Transactions on Information Theory, vol. 49, no. 12, pp. 32133224, Dec 2003.

[11] "SX1272 from Semtech, datasheet," http://www.semtech.com/ wireless-rf/rf-transceivers/sx1272/, accessed: June 16, 2016.

[12] Y. Roth, J.-B. Dore, L. Ros, and V. Berg, "A Comparison of Physical Layers for Low Power Wide Area Networks," in 11th EAI International Conference on Cognitive Radio Oriented Wireless Networks (Crowncom), June 2016.

[13] Y.-J. Wu and L. Ping, "On the limiting performance of turbo-Hadamard codes," IEEE Communications Letters, vol. 8, no. 7, pp. 449-451, July 2004.

[14] S. ten Brink, "Convergence behavior of iteratively decoded parallel concatenated codes," IEEE Transactions on Communications, vol. 49, no. 10 , pp. 1727-1737, Oct 2001.

[15] _ "Convergence of multidimensional iterative decoding schemes," in Conference Record of the Thirty-Fifth Asilomar Conference on Signals, Systems and Computers, vol. 1, Nov 2001, pp. 270-274 vol.1.

[16] L. Bahl, J. Cocke, F. Jelinek, and J. Raviv, "Optimal decoding of linear codes for minimizing symbol error rate (corresp.)," IEEE Transactions on Information Theory, vol. 20, no. 2, pp. 284-287, Mar 1974. 